\title{
Hábitos de alimentación, actividad física y horas de sueño en escolares: un estudio diagnóstico en Educación Primaria
}

\section{Eating habits, physical activity and hours of sleep in school children: a diagnostic study in Primary Education}

\author{
Angustias González RodríGuez ${ }^{1}$ \\ angustias.gonzalez@denf.uhu.es \\ Gabriel (H) Travé González \\ gabriel.trave@dedu.uhu.es \\ Francisca Maria Garcia Padilla \\ fmgarcia@denf.uhu.es \\ Universidad de Huelva, España
}

\section{Resumen:}

El estudio que se presenta tiene una finalidad diagnóstica en relación a los hábitos de vida y alimentación de un grupo de escolares. Se persigue conocer los patrones alimentarios (desayuno y merienda escolar), actividad física y horas de sueño en dos aulas de tercero de Educación Primaria de un centro andaluz. El estudio utiliza una metodología de corte cuantitativo, a partir de un cuestionario administrado a 46 alumnos-as obteniéndose 410 respuestas a lo largo de dos semanas. Los resultados revelan que la práctica totalidad del alumnado ingiere algún alimento antes de acudir al centro escolar, si bien la composición nutricional del mismo es insuficiente. Aproximadamente siete de cada diez alumnos-as expresan la necesidad de ingerir alimento en

\begin{abstract}
:
The present study has a diagnostic purpose in relation to the eating habits and $\mathrm{li}$ festyle of a group of schoolchildren, once symptoms of potential imbalances have been detected by the teaching staff. Eating patterns (breakfast and school afternoon snack), physical activity and rest levels are studied in two third-year Primary Education classrooms in an Andalusian school. The study uses a quantitative methodology, based on a questionnaire administered to 46 students, obtaining 410 responses over two weeks. The results reveal that almost all students eat some food before going to school, although its nutritional composition is limited. Approximately seven out of ten students reported they were hungry while answering the question-
\end{abstract}

1 Dirección para correspondencia (correspondence address):

Angustias González Rodríguez. Departamento de Enfermería. Universidad de Huelva.

Avenida 3 de Marzo. Campus del Carmen, 21071. Huelva (España). 
Hábitos de alimentación, actividad física y horas de sueño en escolares: un estudio diagnóstico en Educación Primaria

Angustias González Rodríguez, Gabriel (H) Travé González y Francisca Maria Garcia

PADILLA

el momento de responder al cuestionario, una hora antes del recreo. Analizando los ingredientes que componen la merienda escolar destacan los zumos y batidos envasados, bocadillos de embutidos y galletas. El análisis de la dimensión de actividad física revela un escenario en el que la muestra se divide equitativamente en función de sus hábitos, predominando no obstante actividades de corte sedentario. Mientras cinco de cada diez alumnos-as no realizan actividad física por las tardes, la mayoría dedica un tiempo considerable a ver la televisión. Finalmente, el análisis de las horas de sueño evidencia que el seis de cada diez alumnos-as duermen una media de 9,2 h., situándose así en el rango mínimo recomendado para esta edad. Una vez finalizado el diagnóstico se realizan propuestas de intervención en el marco del enfoque comunitario.

\section{Palabras clave:}

Hábitos alimentarios; actividad física; desayuno; merienda escolar; Educación Primaria; hábitos saludables. naire, one hour before the school break. As for the ingredients that make up the school snack, they include packaged juices and smoothies, cold cut sandwiches and cookies, with a marginal presence of fruit. The analysis of the physical activity dimension reveals that sedentary activities predominate slightly. While five out of ten students do not do physical activity in the afternoons, the majority spend considerable time watching television. Finally, the analysis of sleep hours shows that six out of ten students sleep an average of 9.2 hours, just within the minimum recommended range. Finally, intervention proposals are made within the framework of the community-led approach.

\section{Key words:}

Eating habits; physical activity; breakfast; school snack; Primary Education; healthy habits.

\section{Résumé:}

L'étude suivante a pour objectif $d^{\prime}$ apporter un diagnostic en ce qui concerne les modes de vie et I'alimentation d'un groupe d'écoliers. Spécifiquement, l'objectif visé est de faire connaître les habitudes d'activité physique, de repos, Id petit déjeuner et de goûter dans deux salles de classe de CE2 dans un établissement scolaire andalou. Cette étude utilise une méthodologie quantitative sur la base d'un questionnaire adressé à quarantesix élèves, permettant d'obtenir 410 réponses, au cours de deux semaines. Les résultats obtenus révèlent que la plupart des élèves consomment quelques aliments avant d'entrer à l'école ; par contre, leur composition nutritionnelle reste insuffisante. Cela conditionne sans aucun doute le fait qu'environ sept élèves sur dix expriment le besoin d'ingérer quelque chose au moment de la réalisation du questionnaire, justement une heure avant la récréation. En analysant les ingrédients qui constituent le repas scolaire, l'accent a été mis sur les jus et les Milkshakes emballés, les sandwichs à la charcuterie et les biscuits. L'analyse de l'activité physique révèle que l'échantillon est divisé également en fonction de leurs habitudes, bien que les activités sédentaires prédominent. Tandis que cinq élèves sur dix n'exercent pas une activité physique le soir, la plupart consacre un temps considérable à regarder la télévision. Enfin, l'analyse des heures de sommeil montre que six élèves sur dix dorment en moyenne 9,2 h, ce qui correspond à la fourchette minimale recommandée à cet âge. Une fois le diagnostic finalisé, les propositions d'intervention sont faites dans le cadre de l'approche communautaire.

\section{Mots clés:}

Habitudes alimentaires; activité physique; petit-déjeuner; goûter scolaire; éducation primaire; habitudes saines.

\section{Fecha de recepción: 28-11-2017 \\ Fecha de aceptación: 1-3-2018}




\section{Introducción}

En la niñez, la adquisición de hábitos de vida saludable constituye una cuestión crítica por su repercusión para la salud actual y futura, siendo crucial el protagonismo de la educación en este ámbito (Varela y Belmonte, 2015). Como sabemos, se está produciendo un alejamiento creciente de la dieta mediterránea al tiempo que los niveles de actividad física están amenazados por múltiples actividades sedentarias (Calleja et al., 2011; Food and Agriculture Organization [FAO], 2008). Así pues, la Organización Mundial de la Salud (OMS, 2017) cuantificaba en 340 miIlones el número de niños-as y adolescentes (5 a 19 años) con sobrepeso u obesidad en el año 2016.

En el complejo entramado de recomendaciones alimentarias, la comunidad científica ha prestado atención especial al desayuno, pues desempeña una función destacada en la alimentación infantil y juvenil (Alsharairi y Somerset, 2016; Aranceta, 2008; Jaúregui-Lobera, 2011; Serra y Aranceta, 2004). Cualitativamente, se considera una de las comidas más importantes por sus múltiples y complejas interacciones con la salud y la educación (Galiano y Moreno, 2010; Haapala et al., 2017). Durante la infancia, la adopción de un modelo incorrecto de desayuno se asocia a un bajo rendimiento físico e intelectual, así como a múltiples problemas de salud (Matthys, De Henauw, Bellemans, De Maeyer, \& De Backer, 2007), siendo especialmente crucial en los sectores de alumnado cuyo estado nutricional presenta déficits (Hoyland, Dye, y Lawton, 2009). Los niños y niñas que van a la escuela sin desayunar, o tomando un desayuno ligero, tienen menor capacidad de atención que aquellos que han realizado un desayuno completo (Adolphus, Lawton, Champ, \& Dye, 2016; Basch, 2011). Fruto de la investigación en este campo, se ha llegado a perfilar un modelo de desayuno que orienta las directrices hacia la promoción de prácticas saludables. De este modo, un desayuno completo se compone de lácteos, cereales y fruta, asegurando la calidad nutricional de esta comida (Dapcich, et al. 2004; Uzhova et al., 2017). Rampersaud, Pereira, Girard, Adams, y Metzl (2005) establecieron las siguientes recomendaciones para los hábitos de desayuno en la población escolar a partir de la revisión de la producción científica sobre el desayuno en niños-as y adolescentes:

- Consumir un desayuno saludable adaptado a las necesidades energéticas diarias. 
Hábitos de alimentación, actividad física y horas de sueño en escolares: un estudio diagnóstico en Educación Primaria

Angustias González Rodríguez, Gabriel (H) Travé González y Francisca Maria Garcia PADILLA

- Ingerir suficiente variedad de grupos de alimentos que aseguren el aporte de micronutrientes y fibra (cereales integrales, frutas y productos lácteos).

- Elegir lácteos bajos en grasa, a excepción de niños y niñas entre 1 y 2 años de edad, que deben consumir leche entera.

- Tomar el desayuno siempre, ya sea en el hogar, en la escuela o en el trayecto.

Con respecto a las recomendaciones para la merienda escolar, no existe una propuesta cerrada de alimentos, al ser la variedad de fuentes alimentarias una garantía de calidad de la dieta omitiendo el consumo de productos alimentarios de baja o nula calidad nutricional (chucherías, dulces, fritos y refrescos, entre otros). Frente a éstos, opciones saludables para la comida de media mañana estarían representadas por la fruta fresca y el yogurt (Ministerio de Sanidad, Política Social e Igualdad, 2010).

Complementariamente a las recomendaciones en torno al desayuno y la merienda escolar, la actividad física es un factor determinante en el mantenimiento del equilibrio nutricional (Katzmarzyk et al., 2015; Martínez Sabater et al., 2013; Poitras et al., 2016). Los beneficios de mantener una vida activa, más allá de la prevención de enfermedades, abarcan el nivel físico, psíquico y socioafectivo, mejorando "las funciones cardiorrespiratorias, la buena forma muscular, la salud ósea y los biomarcadores cardiovasculares y metabólicos" (OMS, 2010, p. 20), además de prevenir el exceso de grasa. Asimismo, diversas fuentes relacionan positivamente la realización de actividad física regular con el rendimiento académico (Donnelly et al., 2016; Marques, Gómez, Martins, Catunda, \& Sarmento, 2017). Por su contribución al equilibrio nutricional y al mantenimiento de un índice de masa corporal adecuado en todas las edades, la práctica de actividad física debe realizarse como mínimo treinta minutos casi todos los días de la semana y con intensidad media (O’Donovan et al., 2010). En la etapa escolar, se recomienda realizar -como mínimo- sesenta minutos diarios de actividad física aeróbica moderada, incorporando otras más vigorosas tres veces a la semana (OMS, 2010; Moreno, Gracia y CNAEP, 2012).

Por lo que respecta a las horas de sueño entre los seis y los diez años, el avance en la maduración del sistema nervioso central determina que la recomendación se encuentre situada en diez horas (Ministerio de Sanidad, Política Social e Igualdad, 2011). La consolidación de hábitos de exposición prolongada a la televisión y el uso de dispositivos móvi- 
les y videojuegos, específicamente en los periodos previos a la hora de acostarse, se relacionan con la alteración del sueño (Greever, Ahmadi, Sirard, \& Alhassan, 2017). No en vano, la falta o mala calidad del sueño ejerce una repercusión en el desarrollo cognitivo y físico de los niños-as (Sánchez-Carpintero, 2008), existiendo evidencias sólidas de su impacto en el aumento de la obesidad (Chaput, 2016).

En España, los temas relacionados con la salud se han considerado tradicionalmente responsabilidad casi exclusiva del ámbito sanitario. No obstante, diferentes investigaciones han evidenciado la relevancia de los centros educativos en este campo (Langford et al., 2011;Schwartz, Leardo, Aneja, \& Elbel, 2016), así como la importancia de la formación del profesorado en la Promoción de la Salud ya desde sus fases iniciales (Torres-García y Santana-Hernández, 2017).

Situados en el contexto educativo andaluz, desde un centro escolar se detectan señales que indican deficiencias en los hábitos de vida del alumnado de tercer curso de Educación Primaria. De este modo, los niños-as expresan la necesidad de comer en las primeras franjas del horario escolar, mostrando además síntomas de cansancio y falta de atención. Ante esta problemática, se plantean los siguientes interrogantes:

- ¿El alumnado desayuna adecuadamente antes de ir al colegio?

- ¿La elección de la merienda escolar es la más deseable?

- ¿Qué hábitos de actividad física realiza el alumnado?, y ¿cuántas horas de sueño se computan?

Para dar respuesta a estas preguntas, se lleva a cabo un estudio en función de los siguientes objetivos: a) conocer las prácticas alimentarias en torno al desayuno y merienda escolar, y b) conocer los hábitos de actividad física y horas de sueño del alumnado.

\section{Diseño}

El estudio se articuló a través de la administración de un cuestionario al alumnado de tercero de Educación Primaria de un centro andaluz. Se establecieron dos categorías: 1. Características del desayuno y de la merienda escolar; y 2. Perfil de actividad física y horas de sueño.

El estudio se desarrolló en un centro escolar de una localidad costera de la provincia andaluza de Huelva. La muestra objeto de estudio la 
Hábitos de alimentación, actividad física y horas de sueño en escolares: un estudio diagnóstico en Educación Primaria

Angustias González Rodríguez, Gabriel (H) Travé González y Francisca Maria Garcia PADILLA

constituyeron 44 niños y niñas, 24 en cada clase, con una edad media de ocho años. Globalmente se computaban más niñas que niños (tabla 1).

Tabla 1. Sexo del alumnado participante y distribución por aulas.

\begin{tabular}{cccc}
\hline Alumnado & Aula $3^{\circ} \mathrm{A}$ & Aula $3^{\circ} \mathrm{B}$ & Total \\
\hline Niños & 11 & 10 & 21 \\
Niñas & 13 & 14 & 27 \\
Total & 24 & 24 & 48 \\
\hline
\end{tabular}

Se ha utilizado un cuestionario autoadministrable validado en el contexto español (Banet y López, 2010), al que se le añadieron contenidos relacionados con la merienda escolar. El cuestionario recogía información sobre los hábitos alimentarios, la actividad física y las horas de sueño nocturno del alumnado. La estructura final del instrumento se compuso de seis variables, desarrolladas en nueve preguntas de opción múltiple (tabla 2).

Tabla 2. Categorías, subcategorías e ítems del cuestionario AFDDyME-alumnado.

\begin{tabular}{|c|c|c|}
\hline Variables & Subvariables & Preguntas \\
\hline $\begin{array}{l}\text { I. Datos de } \\
\text { identificación }\end{array}$ & $\begin{array}{l}\text { Nombre y apellidos, fecha y hora del } \\
\text { registro. }\end{array}$ & \\
\hline $\begin{array}{l}\text { II. Actividad } \\
\text { física y ocio }\end{array}$ & $\begin{array}{l}\text { Tipo de actividad, física y/o intelectual, } \\
\text { realizada la tarde anterior al registro. }\end{array}$ & $\begin{array}{l}\text { 1. ¿Qué actividades } \\
\text { realizaste ayer? }\end{array}$ \\
\hline $\begin{array}{l}\text { III. Horas de } \\
\text { sueño noc- } \\
\text { turno }\end{array}$ & $\begin{array}{l}\text { Horario de dormir y de levantarse el } \\
\text { día del registro. }\end{array}$ & $\begin{array}{l}\text { 2. ¿A qué hora te acos- } \\
\text { taste anoche? } \\
\text { 3. ¿A qué hora te le- } \\
\text { vantaste ayer? }\end{array}$ \\
\hline \multirow{2}{*}{$\begin{array}{l}\text { IV. Tipo de } \\
\text { desayuno } \\
\text { realizado en } \\
\text { casa }\end{array}$} & Ingesta de alimento sólido. & $\begin{array}{l}\text { 4. ¿Qué has comido } \\
\text { en el desayuno? }\end{array}$ \\
\hline & Ingesta de alimento líquido. & $\begin{array}{l}\text { 5. ¿Qué has bebido en } \\
\text { el desayuno? }\end{array}$ \\
\hline
\end{tabular}

\section{Otras} pautas ali- Descripción del desayuno: duración,
mentarias en relación al apetencia antes de desayunar. desayuno

6. ¿Cómo has realizado tu desayuno?

7. ¿Tenías ganas de desayunar?

VI. Tipo de merienda escolar
8. ¿Ahora tienes ganas

Ganas de comer en el momento de re- de comer? llenar el cuestionario.

9. ¿Qué has traído Tipo de alimento elegido para el recreo. para la merienda del colegio? 
El cuestionario fue administrado al alumnado en el aula entre las $10.30 \mathrm{~h}$ y las $11.00 \mathrm{~h}$ de la mañana. En el aula A se realizó en las primeras horas de la jornada escolar, antes de las $11.00 \mathrm{~h}$. Por el contrario, en el aula B se eligieron diferentes horarios, siendo frecuente los días que se registró a partir de las $11.30 \mathrm{~h}$. De los cuestionarios realizados después del recreo, se ha excluido del análisis el apartado que preguntaba sobre las ganas de comer o beber debido a la incidencia de la merienda escolar. El alumnado cumplimentó el cuestionario periódicamente durante el desarrollo de la investigación, formando parte de la rutina escolar. Se recogieron un total de 410 cuestionarios, 178 en el aula A en ocho días y 232 en el aula B durante diez días.

El tratamiento de los datos se realizó a través de la base de datos SPSS $®$ v. 19, aplicándose medidas de estadística descriptiva (frecuencias y porcentajes).

El análisis del desayuno se articuló a través de la propuesta de Banet y López (2010), incorporando algunas variantes. Así, se establecieron tres modelos de desayuno (completo, incompleto y de baja calidad nutricional) y cinco tipologías (figura 1).

\begin{tabular}{|c|c|}
\hline $\begin{array}{c}\text { Modelo } 1 \\
\text { Desayuno completo }\end{array}$ & $\begin{array}{l}\text { Tipo 1: integrado por un lácteo, cereales (copos o pan), fruta o } \\
\text { zumo natural y acompañamiento graso de calidad (aceite de oliva } \\
\text { o alimentos bajos en grasas en el que se excluyen grasas trans). }\end{array}$ \\
\hline 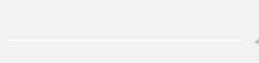 & $\begin{array}{l}\text { Tipo 2: se compone de dos grupos de alimentos, uno de ellos es } \\
\text { fruta o zumo natural. }\end{array}$ \\
\hline $\begin{array}{l}\text { Modelo } 2 \\
\text { Desayuno incompleto }\end{array}$ & $\begin{array}{l}\text { Tipo 3: combina alimentos a partir de cereales y leche (pan, } \\
\text { cereal en copos con leche sola o chocolateada). } \\
\text { Tipo 4: el de menor aporte energético del grupo. Compuesto } \\
\text { exclusivamente de un alimento líquido o sólido (leche, leche con }\end{array}$ \\
\hline Modelo 3 & cacao, pan dulce...). \\
\hline $\begin{array}{l}\text { Desayuno de baja } \\
\text { calidad nutricional }\end{array}$ & $\begin{array}{l}\text { Tipo 5: compuesto por bollería, pastelería industrial, zumo } \\
\text { comercial; estando ausente la leche, la fruta fresca entera o zumo } \\
\text { natural. }\end{array}$ \\
\hline
\end{tabular}

Figura 1. Clasificación de los diferentes tipos de desayuno.

El desayuno completo nutricionalmente es considerado adecuado en función de las recomendaciones rubricadas desde la comunidad científica (Ministerio de Sanidad y Política Social, y Ministerio de Educación, 2007). En el desayuno incompleto el aporte de energía y nutrientes se encuentra por debajo del anterior al carecer de uno o varios alimentos en su composición. Por último, el desayuno de baja calidad nutricional incluye productos no recomendados en la dieta habitual de escolares, aunque su aporte energético pueda ser aceptable. 
Hábitos de alimentación, actividad física y horas de sueño en escolares: un estudio diagnóstico en Educación Primaria

Angustias González Rodríguez, Gabriel (H) Travé González y Francisca Maria Garcia PADILLA

\section{Resultados}

\subsection{Categoría 1: Características del desayuno y la merienda escolar}

La descripción y análisis de las características del desayuno y la merienda escolar se exponen a continuación.

\subsubsection{TIPO DE DESAYUNO}

Si analizamos los resultados globalmente, el alumnado que va al colegio sin ingerir ningún alimento es bastante reducido. El porcentaje promedio de alumnos-as de ambas aulas que no desayuna se sitúa en el 3.9\%.

A partir de estas cifras, encontramos una tasa considerable de niños y niñas que toman algún alimento antes de acudir al centro escolar. Aunque la mayoría del alumnado (96.1\%) afirme comer o beber algo, es necesario analizar las características de la ingesta y determinar su calidad nutricional. Los tipos de desayuno señalados por el alumnado participante se exponen en la tabla 3.

Tabla 3. Porcentaje global de ingesta de desayuno y tipología de desayuno en cada aula.

\begin{tabular}{|c|c|c|c|c|c|}
\hline $\begin{array}{c}\text { Alumnado que } \\
\text { no desayuna }\end{array}$ & \multicolumn{5}{|c|}{ Alumnado que desayuna } \\
\hline \multirow[t]{3}{*}{3.9} & & & 96.1 & & \\
\hline & \multicolumn{5}{|c|}{ Tipos de desayuno } \\
\hline & Completo & & Incompletc & & $\begin{array}{c}\text { Baja calidad } \\
\text { nutricional }\end{array}$ \\
\hline Aulas & Tipo 1 & Tipo 2 & Tipo 3 & Tipo 4 & Tipo 5 \\
\hline Aula A & 2.2 & 4.4 & 58.1 & 25.2 & 6.6 \\
\hline Aula B & 4.8 & 2.9 & 56.2 & 28 & 3.4 \\
\hline Todos & 3.8 & 3.2 & 57 & 27.1 & 4.7 \\
\hline
\end{tabular}

Como podemos observar, el desayuno completo -que sirve de modelo de desayuno óptimo por incluir cereal, lácteo y fruta- es minoritario (3.8\%). La ingesta más frecuente (57\%) se corresponde con el desayuno incompleto tipo 3, integrado por pan o cereal en copos y leche. El desayuno incompleto tipo 4, basado exclusivamente en un alimento líquido, es el consumido por más de la cuarta parte del grupo $(27.1 \%)$. Por último, el desayuno definido de baja calidad nutricional -por incluir productos no recomendados- es señalado por un número reducido de alumnos-as (4.7\%). 
El grupo de alimentos más frecuente en ambas aulas es el cereal y sus derivados; por el contrario, el consumo global de fruta es poco habitual $(1.7 \%)$. En la misma línea, el consumo de dulces, representa una ingesta minoritaria ( $2.6 \%$ en el aula A y $0.9 \%$ en el aula B). En los casos en que se consumen, suelen ser -en orden de mayor a menor frecuencia- magdalenas, donuts, palmeras, sobaos y tortitas. En el grupo de alumnos-as del aula B se observa que el alumnado que toma dos o más tostadas es significativamente mayor (14.9\% frente a $5.3 \%$ ) (tabla 4 ).

Tabla 4. Porcentaje de los alimentos sólidos ingeridos en el desayuno.

\begin{tabular}{lccc}
\hline \multirow{2}{*}{ Alimentos sólidos } & \multicolumn{3}{c}{ Aulas } \\
\cline { 2 - 4 } & Aula A & Aula B & Promedio \\
\hline Una tostada & 22.7 & 22 & 22.3 \\
Dos o más tostadas & 5.3 & 14.9 & 10.1 \\
Cereal y leche & 10.9 & 13.9 & 12.4 \\
Galletas & 21.1 & 10.8 & 15.9 \\
Fruta entera & 2.6 & 0.9 & 1.7 \\
Dulce casero & - & 2.3 & 1.1 \\
Otra cosa & 7.1 & 9.7 & 8.4 \\
Nada & 29.9 & 25 & 27.4 \\
\hline
\end{tabular}

En cuanto a la ingesta líquida del desayuno, la bebida mayoritaria es la leche con cacao $(66.1 \%)$, seguida por la leche sola (13.9) (tabla 5).

Tabla 5. Porcentaje promedio de alimentos líquidos ingeridos en el desayuno.

\begin{tabular}{lccc}
\hline Tipología de alimentos líquidos & Aula A & Aula B & Promedio \\
\hline Leche sola & 15.4 & 12.4 & 13.9 \\
Leche con cacao & 59.2 & 73 & 66.1 \\
Zumo casero & 3.4 & 2.4 & 2.9 \\
Zumo envasado & 2.1 & 1.4 & 1.75 \\
Café con leche & 6 & 1.4 & 3.7 \\
Batido casero & - & 0.5 & .25 \\
Batido envasado & 4.2 & - & 2.1 \\
Otra cosa & 0.6 & 3.3 & 1.95 \\
Nada & 8.7 & 6 & 7.35 \\
\hline
\end{tabular}


Hábitos de alimentación, actividad física y horas de sueño en escolares: un estudio diagnóstico en Educación Primaria

Angustias González Rodríguez, Gabriel (H) Travé González y Francisca Maria Garcia PADILLA

\subsubsection{Forma DE REALIZAR EL DESAYUNO Y ACOMPAÑAMIENTO}

Aludiendo al modo en cómo se realiza el desayuno, predomina el alumnado que lo toma sentado ( $83 \%$ en ambas clases). Por otra parte, en cuanto al acompañamiento, del $53.6 \%$ de alumnado que desayuna en compañía de uno de sus familiares, la presencia de la madre y hermanos-as es la pauta más frecuente (tabla 6).

Tabla 6. Porcentaje promedio de la forma de realizar el desayuno y acompañamiento.

\begin{tabular}{|c|c|c|c|c|c|}
\hline \multicolumn{5}{|c|}{ Sentado } & De pie \\
\hline & & $83 \%$ & & & $17 \%$ \\
\hline $\begin{array}{l}\text { Sin com- } \\
\text { pañía }\end{array}$ & Madre & Padre & Hermano-a & Abuela & $\begin{array}{l}\text { Dos o más familiares (madre } \\
\text { y padre, más hermanos-as) }\end{array}$ \\
\hline 44.4 & 15.1 & 3.6 & 12.6 & 4 & 18.3 \\
\hline
\end{tabular}

3.1.3 NeCesidad De COMER el Desayuno en CASA y en el mOMENTO DeL REGISTRO

Cuando se pregunta al alumnado sobre las ganas que tenía de desayunar en su casa, más de la mitad expresa su apetencia (58,6\%). Este porcentaje aumenta al preguntarles sobre la necesidad de comer en el momento del registro, con una mayoría de alumnado que reconoce tener apetito en el momento de contestar al cuestionario (siete de cada diez niños-as en ambas clases) (tabla 7).

Tabla 7. Porcentaje promedio de las ganas de realizar el desayuno en casa en ambos grupos.

\begin{tabular}{cccc}
\hline & $\begin{array}{c}\text { Con ganas } \\
\text { de comer }\end{array}$ & $\begin{array}{c}\text { Sin ganas } \\
\text { de comer }\end{array}$ & $\begin{array}{c}\text { No } \\
\text { contesta }\end{array}$ \\
\hline Antes del desayuno en casa & 33,7 & 58,6 & 7,6 \\
\hline En el momento de rellenar el cuestionario & 20.1 & 68,8 & 11.4 \\
\hline
\end{tabular}

\subsubsection{CARACTERÍ́STICAS DE LA MERIENDA ESCOLAR}

Los resultados correspondientes a la pregunta ¿Qué te has traído para la merienda del colegio? se presentan separados por aulas. En cuanto a la merienda del alumnado del aula A, observamos que las bebidas mayoritarias son el zumo y el batido envasado (figura 4). Los alimentos sólidos principales son bocadillos y galletas; mientras que el consumo de fruta es muy reducido, el del dulce casero es inexistente. 
Hábitos de alimentación, actividad física y horas de sueño en escolares: un estudio diagnóstico en Educación Primaria

angustias González Rodríguez, Gabriel (H) Travé González y Francisca Maria Garcia PADILLA

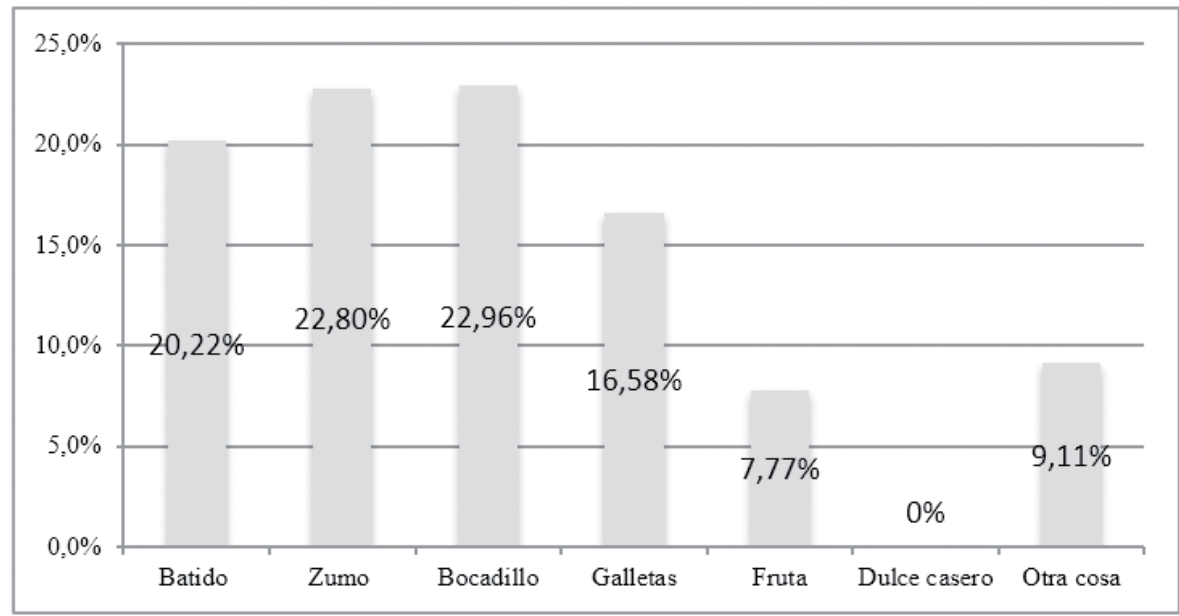

Figura 4. Porcentaje promedio de alimentos elegidos para la merienda escolar en aula A.

En cuanto a la merienda del aula $B$, en el análisis se observa que el repertorio de bebidas elegidas es coincidente con el grupo anterior: batido y zumo envasado, siendo el consumo de agua minoritario en ambas aulas. El alimento sólido principal es el bocadillo (figura 5).

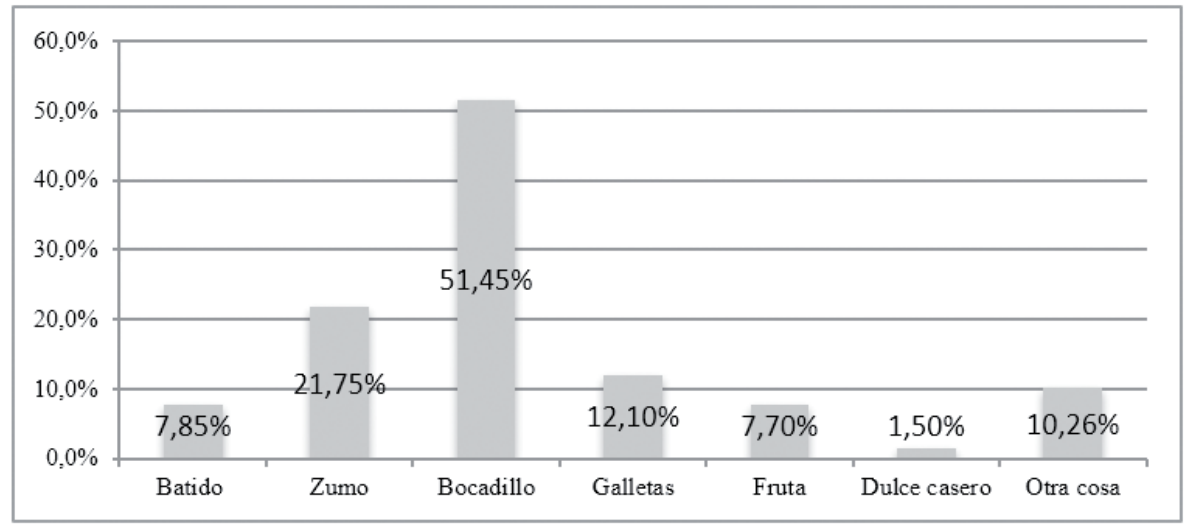

Figura 5. Porcentaje promedio de alimentos elegidos para la merienda escolar en aula B.

Finalmente, el análisis del repertorio de alimentos que acompañan al pan del bocadillo para ambas clases revela la predominancia de los embutidos (figura 6). 
Hábitos de alimentación, actividad física y horas de sueño en escolares: un estudio diagnóstico en Educación Primaria

Angustias González Rodríguez, Gabriel (H) Travé González y Francisca Maria Garcia PADILLA

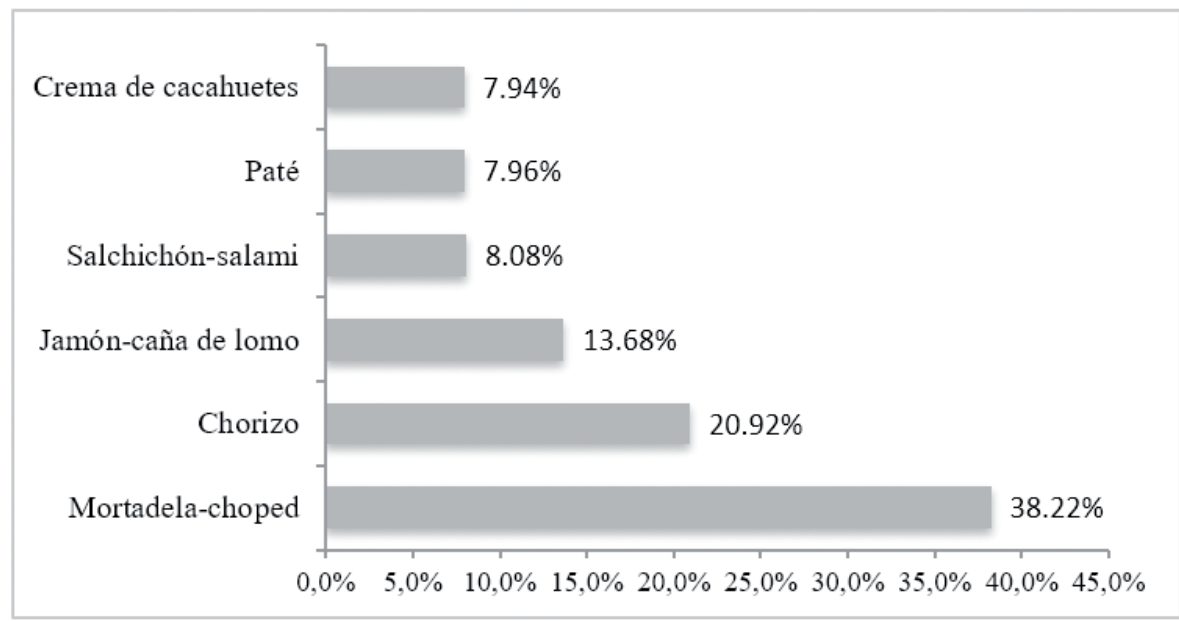

Figura 6. Porcentaje promedio del repertorio de acompañamiento del bocadillo de la merienda escolar.

\subsection{Categoría 2: Perfil de actividad física y descanso}

Para facilitar el análisis, los resultados en torno a las actividades realizadas la tarde anterior se han agrupado en cinco tipologías (sedentarias en casa, juegos activos fuera de casa, actividades deportivas, actividades combinadas y otras actividades) (tabla 8). Los datos indican que el $43.2 \%$ del alumnado realiza habitualmente actividades sedentarias. Con todo, es ligeramente superior el alumnado que combina distintas actividades $(46.1 \%)$.

Tabla 8. Porcentaje promedio del tipo de actividad realizada la tarde anterior al registro.

\begin{tabular}{ccccc}
\hline \multicolumn{5}{c|}{ ¿Qué actividades realizaste ayer? } \\
\hline $\begin{array}{c}\text { Actividades se- } \\
\text { dentarias en casa }\end{array}$ & $\begin{array}{l}\text { Juegos activos } \\
\text { fuera de casa }\end{array}$ & $\begin{array}{c}\text { Actividades } \\
\text { deportivas }\end{array}$ & $\begin{array}{c}\text { Actividades } \\
\text { combinadas }\end{array}$ & $\begin{array}{c}\text { Otras } \\
\text { actividades }\end{array}$ \\
\hline 43.2 & 4.2 & 4.6 & 46.1 & 1.7 \\
\hline
\end{tabular}

Analizados los registros en el conjunto de actividades sedentarias, se confirma que las prácticas más frecuentes entre el alumnado se relacionan con ver televisión (52.9\%), leer (24.8\%), hacer deberes $(12.7 \%)$ y jugar a videojuegos $(9.5 \%)$; frente a prácticas activas diversas como realizar deporte $(22.1 \%)$ y jugar en la calle $(18.5 \%)$. 
Finalmente, abordando las pautas de descanso nocturno, la media de horas de sueño del alumnado se sitúa en 9,22 h. con una desviación típica de 0,92. El histograma de la figura 7 muestra que un 35\% de los niños-as duerme un número de horas inferior a la media, mientras que el resto (65\%) descansa 9,30 h. o más (figura 7).

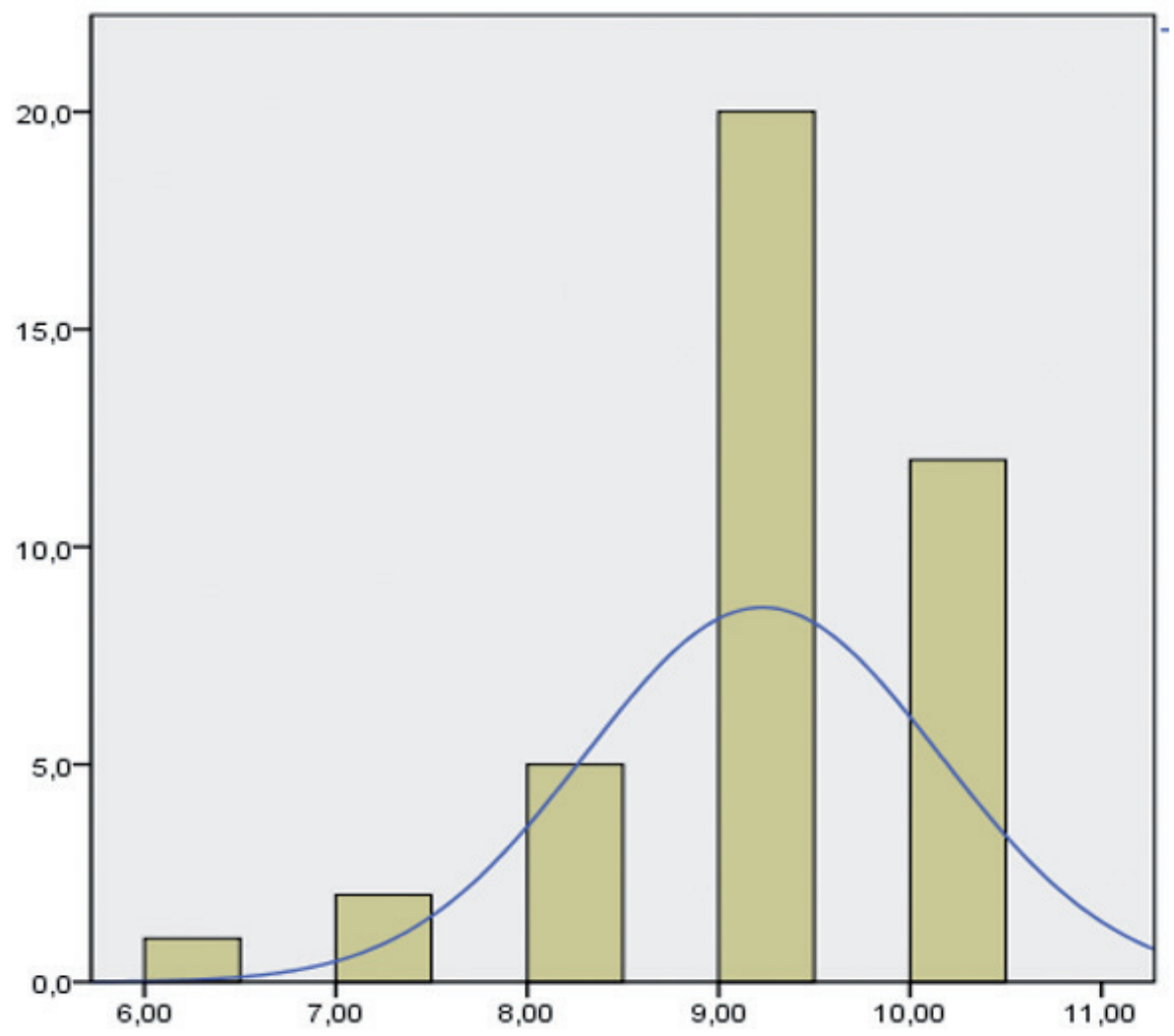

Figura 7. Histograma con la media de las horas de sueño del alumnado.

\section{Discusión y conclusiones}

Los resultados del estudio evidencian que solo una minoría del alumnado participante realiza un desayuno completo, principalmente por el escaso consumo de fruta. Con respecto a los alimentos que con más frecuencia componen la merienda escolar, se encuentran los zumos y batidos como ingesta líquida y, dentro de los sólidos, los bocadillos con 
distintos rellenos. Por lo que se refiere a los niveles de actividad física, se detectan hábitos de vida sedentarios entre el alumnado con una clara división equitativa de la muestra. Prestando atención a las horas de sueño nocturno, se pone de relieve que la mayor parte del alumnado se sitúa en el umbral mínimo recomendado.

Seguidamente abordamos las conclusiones finales en función de los objetivos planteados en el estudio.

\section{Desayuno y merienda escolar}

Analizando los resultados relativos al desayuno, prácticamente la totalidad del alumnado ingiere algún alimento antes de acudir al centro escolar, hecho coincidente con diferentes estudios realizados en el contexto español (Cubero et al., 2014; Díaz, Ficapa-Cusí, y Aguilar-Martínez, 2016; Menal, Fajó, y Marques, 2011; Molinero et al., 2010; Muñoz-Carrillo, Ruiz-Díaz, Hernández-Ruiz, y Córdoba-Ruiz, 2013; Tovar-Gálvez, Martín-Cuesta, González-Jiménez, y Schmidt-RioValle, 2017). Además, se pone de manifiesto que una mayoría del alumnado manifiesta sentir ganas de realizar el desayuno en casa. Profundizando en las características del desayuno, predominan las modalidades incompletas: principalmente el tipo 3, basado en pan o cereal en copos y leche; y, el tipo 4 compuesto por un solo elemento líquido o sólido. En consecuencia, ocho de cada diez alumnos-as realizan un desayuno que se aleja de las características energéticas y nutricionales del desayuno óptimo y de calidad (Ministerio de Sanidad y Consumo, 2005). Esta configuración alimentaria difícilmente cubriría el valor calórico diario recomendado para esta comida, que se sitúa en el 20\% (Galiano y Moreno, 2010), alcanzando el 30\% si incluye la merienda escolar (Ministerio de Sanidad y Política Social, Ministerio de Educación, 2007). Un síntoma de la insuficiente cantidad ingerida se evidencia ante el hecho de que el alumnado de ambas aulas exprese necesidad de ingerir alimento en el momento en que se administra el cuestionario. De especial relevancia resulta que el $69 \%$ del alumnado del aula A manifieste ganas de comer o beber antes de las 11.00, considerando que la merienda escolar se realiza a partir de las 12:00. Nuestro contexto cultural representa, en sí mismo, un limitador a la hora de calibrar la relevancia de hacer un desayuno suficiente y saludable. De hecho, un reciente estudio ha confirmado la generalización de los desayunos insuficientes entre población adulta española 
(estimado en menos del 5\% de la ingesta total del día), relacionándose esta práctica con el riesgo de la aparición temprana de arterioesclerosis y otras patologías (Uzhova et al., 2017).

La presencia de la fruta en el desayuno es marginal, si bien su consumo es considerado necesario para un desayuno óptimo conjuntamente con al menos un lácteo y un cereal (Agencia Española de Consumo, Seguridad Alimentaria y Nutrición [AECOSAN], 2016). Por su parte, las condiciones en las que se realiza el desayuno reflejan una práctica mayoritaria de tomarlo sentado (ocho de cada diez niños-as), estando principalmente acompañados de la madre y hermanos-as. En cuanto a los alimentos que componen la merienda escolar son principalmente zumos y batidos envasados, bocadillos de embutidos y galletas. Por el contrario, el consumo de fruta es muy reducido, situación similar a la encontrada en otros estudios (González-González, Falero-Gallego, Redondo-González, y Muñoz-Serrano, 2016; Muñoz, Fernández y Navarro, 2015). La ingesta de dulces, bollería industrial y chucherías en la merienda escolar es anecdótica.

\section{Actividad física}

El análisis de los registros realizados por el alumnado en torno a la actividad física revela un escenario en el que la realización exclusiva de actividades sedentarias tiene un peso importante para prácticamente la mitad del alumnado. Menos de la cuarta parte hace deporte y solo un grupo más reducido sale a la calle a jugar, siendo la visualización de televisión la actividad más habitual en el grupo participante. Estos datos son congruentes con los resultados de diversos estudios que han puesto de relieve el exceso de tiempo dedicado a las actividades sedentarias en la infancia (AECOSAN, 2016; OMS, 2017). En concreto, el informe 2016: Actividad Física en niños y adolescentes en España (Fundación para la Investigación Nutricional [FIN], 2016), al analizar el juego activo fuera de la escuela, concluye que la adherencia a esta práctica en la infancia española se encuentra entre el $41 \%$ y el $60 \%$.

\section{Horas de sueño}

Abordando las horas de sueño, seis de cada diez alumnos-as descansan 9,30 h. o más, encontrándose en el límite del margen de seguridad reco- 
mendado para el grupo de edad de entre 6 y 12 años que se sitúa en la horquilla de entre 9 a 12 horas (Paruthi et al., 2016).

Por lo que respecta a las limitaciones del estudio, se pone de manifiesto que la reducida selección de la muestra imposibilita la generalización de los resultados obtenidos, habiendo de interpretarse como el diagnóstico de situación de un contexto específico. Sin embargo, se ha evidenciado cómo éstos son congruentes con el estado de la cuestión en diferentes estudios que han analizado previamente los patrones de alimentación, actividad física y horas de sueño del alumnado en nuestro país; siendo, por tanto, reflejo de una situación habitual ante la que es preciso intervenir (Díaz, Ficapa-Cusí y Aguilar-Martínez 2016; FIN, 2016; Tovar-Gálvez, Martín-Cuesta, González-Jiménez y Schmidt-RioVaIle, 2017;)

Por ello, desde el ámbito escolar es preciso diseñar y desarrollar propuestas didácticas vinculadas con la promoción de hábitos de vida saludable sostenidas en el tiempo, al ser la escuela un ámbito de intervención estratégico (Kastorini, 2016; Leme et al., 2016; Llargués et al., 2011; Waters et al., 2017). Asimismo, se apuesta por el desarrollo de actuaciones dentro del enfoque comunitario que integren los contextos escolar, familiar y social haciendo especial hincapié en la colaboración de otros sectores (Del Campo, Vara, y Navarro, 2010). El desarrollo de proyectos de intervención centrados en el desayuno representaría un buen punto de partida, considerando los efectos comprobados que ejerce un desayuno saludable y completo en el rendimiento cognitivo del alumnado, especialmente en aquellos nutricionalmente vulnerables (Hoyland, Dye, y Lawton, 2009). Se podrían impulsar, dentro del marco de las Redes de Escuelas Promotoras de Salud, acciones encaminadas a "la incorporación del mundo de la gastronomía, como una herramienta eficaz para potenciar hábitos saludables de alimentación en niños; talleres de cocina y del gusto, talleres sobre el desperdicio alimentario, visitas a mercados $y$, si las condiciones lo permiten, desarrollo de huertos escolares" (Varela y Belmonte, 2015, p. 309). Asimismo, sería necesario apoyar el diseño, desarrollo y evaluación de propuestas didácticas de corte funcional enfocadas al tratamiento del hecho alimentario desde enfoques integrados que contribuyan a reelaborar y mejorar tanto las concepciones como las prácticas alimentarias del alumnado y sus familias (Pozuelos, GarcíaPrieto, y Gracia, 2017; Pozuelos, González, y Travé; 2008).

Encontrar puntos de encuentro donde pueda colaborar la escuela, la 
Hábitos de alimentación, actividad física y horas de sueño en escolares: un estudio diagnóstico en Educación Primaria

Angustias González Rodríguez, Gabriel (H) Travé González y Francisca Maria Garcia

PADILLA

familia y los profesionales sanitarios supone adoptar iniciativas emergentes, complejas y que inevitablemente aumentan la carga de trabajo de aquellos involucrados. Por ello, se requieren de compromisos, apoyos y recursos institucionales para hacer frente al reto de garantizar la salud y el bienestar de la infancia.

\section{Referencias}

Adolphus, K., Lawton, C.L., Champ, C.L., \& Dye, L. (2016). The effects of breakfast and breakfast composition on cognition in children and adolescents: a systematic review. Advances in Nutrition: An International Review Journal, 7(3), 590S-612S. doi:10.3945/an.115.010256

Agencia Española de Consumo, Seguridad Alimentaria y Nutrición (AECOSAN) (2016). Estudio ALADINO. Estudio de vigilancia del crecimiento, alimentación, actividad física, desarrollo infantil y obesidad en España. Madrid: Ministerio de Sanidad, Servicios Sociales e Igualdad.

Alsharairi, N.A. \& Somerset, S.M. (2016). Skipping breakfast in early childhood and its associations with maternal and child BMI: a study of 2-5-year-old Australian children. European journal of clinical nutrition, 70(4), 450-455. doi:10.1038/ejcn.2015.184

Aranceta, J. (2008). Obesidad infantil: Nuevos hábitos alimentarios y nuevos riesgos para la salud. Análisis de sus causas. En C. Díaz y C. Gómez (Coords.), Alimentación, consumo y salud. Colección Estudios Sociales, nº 24 (pp. 216-245). Barcelona: Obra Social Fundación "La Caixa".

Banet, E. y López, C. (2010). ¿Cómo mejorar el desayuno de los escolares de educación primaria? Investigación en la Escuela, 71, 63-83.

Basch, C.E. (2011). Breakfast and the achievement gap among urban minority youth. Journal of School Health, 81(10), 635-640. doi:10.1111/j.17461561.2011.00638.x

Calleja, A., Muñoz-Weigand, C., Ballesteros, M.D., Vidal, A., López Gómez, J.J., Cano, I., ...García Fernández, M.C. (2011). Modificación de los hábitos alimentarios del almuerzo en una población escolar. Nutrición Hospitalaria: Órgano Oficial de la Sociedad Española de Nutrición Parenteral y Enteral, 26(3), 560-565. doi:10.3305/ nh.2011.26.3.4692

Chaput, J. P. (2016). Is sleep deprivation a contributor to obesity in children?. Eating and Weight Disorders-Studies on Anorexia, Bulimia and Obesity, 21(1), 5-11. doi:10.1007/s40519-015-0233-9

Cubero, J., Guerra, S., Calderón, M.A., Luengo, L.M., Pozo, A., y Ruiz, C. (2014). Análisis del desayuno escolar en la provincia de Badajoz (España). Rev Esp Nutr Comunitaria, 20(4), 51-56. doi:10.7400/RENC.2014.01.2.5011

Dapcich, V., Salvador, G., Ribas, L., Pérez, C., Aranceta, J., y Serra, L. (2004). Guía de la Alimentación Saludable. Madrid: Sociedad Española de Nutrición Comunitaria (SENC) y Sociedad Española de Medicina de Familia y Comunitaria (semFYC). Re- 
Hábitos de alimentación, actividad física y horas de sueño en escolares: un estudio diagnóstico en Educación Primaria

Angustias González Rodríguez, Gabriel (H) Travé González y Francisca Maria Garcia

PADILLA

cuperado de http://www.semfyc.es/pfw_files/cma/Informacion/modulo/documentos/ guia_alimentacion.pdf

Del Campo, M.L., Vara, M. y Navarro, A. (2010). Educación alimentaria-nutricional (EAN) en la enseñanza primaria municipal de córdoba. Una experiencia en investigación-acción participativa (IAP). Diaeta, 28(132), 15-22. Recuperado de http://www. scielo.org.ar/scielo.php?pid=S1852

Díaz, T., Ficapa-Cusí, P. y Aguilar-Martínez, A. (2016). Hábitos de desayuno en estudiantes de primaria y secundaria: posibilidades para la educación nutricional en la escuela. Nutrición Hospitalaria, 33(4), 909-914. doi:10.20960/nh.391

Donnelly, J.E., Hillman, C.H., Castelli, D., Etnier, J.L., Lee, S., Tomporowski, P., \& SzaboReed, A.N. (2016). Physical activity, fitness, cognitive function, and academic achievement in children: a systematic review. Medicine and science in sports and exercise, 48(6), 1197-1222. doi:10.1249/MSS.0000000000000901

Food and Agriculture Organization [FAO] (2008). The state of food insecurity in the World. High food prices and foodsecurity threats and opportunities. Rome: FAO.

Fundación para la Investigación Nutricional [FIN] (2016). Informe 2016: Actividad Física en niños y adolescentes en España. Fundación para la Investigación Nutricional (FIN): Barcelona. Recuperado de http://www.observatoriodelainfancia.es/oia/esp/documentos_ficha.aspx?id=5056

Galiano, M.J. y Moreno, J.M. (2010). El desayuno en la infancia: más que una buena costumbre. Acta Pediátrica Española, 68(8), 403-408.

González-González, A., Falero-Gallego, M.P., Redondo-González, O. y Muñoz-Serrano, A. (2016). Patrón dietético de la población escolar del Área La Mancha-Centro (Ciudad Real). Anales de Pediatría, Vol 84(3), 133-138. doi:10.1016/j.anpedi.2015.06.001

Greever, C.J., Ahmadi, M., Sirard, J. \& Alhassan, S. (2017). Associations among physical activity, screen time, and sleep in low socioeconomic status urban girls. Preventive Medicine Reports, 5, 275-278. doi:10.1016/j.pmedr.2017.01.014

Haapala, E.A., Eloranta, A.M., Venäläinen, T., Jalkanen, H., Poikkeus, A.M., Ahonen, T., \& Lakka, T.A. (2017). Diet quality and academic achievement: a prospective study among primary school children. European Journal of Nutrition, 56(7), 2299-2308. doi:10.1007/s00394-016-1270-5

Hoyland, A., Dye, L. y Lawton, C.L. (2009). A systematic review of the effect of breakfast on the cognitive performance of children and adolescents. Nutrition research reviews, $22(2), 220-243$.

Jaúregui-Lobera, I. (2011). Desayuno y funciones cognitivas en la infancia y la adolescencia. Una revisión sistemática. Revista Española Nutrición Comunitaria, 17(3), 151-160.

Kastorini, C.M., Lykou, A., Yannakoulia, M., Petralias, A., Riza, E. \& Linos, A. (2016). The influence of a school-based intervention programme regarding adherence to a healthy diet in children and adolescents from disadvantaged areas in Greece: the DIATROFI study. I Epidemiol Community Health, 70(7), 671-677. doi:10.1136/jech2015-205680

Katzmarzyk, P.T., Barreira, T.V., Broyles, S.T., Champagne, C.M., Chaput, J.P., ... Church, T.S. (2015). Relationship between lifestyle behaviors and obesity in children ages 
Hábitos de alimentación, actividad física y horas de sueño en escolares: un estudio diagnóstico en Educación Primaria

Angustias González Rodríguez, Gabriel (H) Travé González y Francisca Maria Garcia

PADILLA

9-11: results from a 12-country study. Obesity, 23(8), 1696-1702. doi:10.1002/ oby. 21152

Langford, R., Campbell, R., Magnus, D., Bonell, C.P., Murphy, S.M., Waters, E., ... Gibbs, L. F. (2011). The WHO Health Promoting School framework for improving the health and well-being of students and staff. Cochrane Database Syst Rev, 1. doi:10.1002/14651858.CD008958.pub2

Leme, A.C.B., Lubans, D.R., Guerra, P.H., Dewar, D., Toassa, E.C. \& Philippi, S.T. (2016). Preventing obesity among Brazilian adolescent girls: Six-month outcomes of the Healthy Habits, Healthy Girls-Brazil school-based randomized controlled trial. Preventive medicine, 86, 77-83. doi:10.1016/j.ypmed.2016.01.020

Llargués, E., Franco, R., Recasens, A., Nadal, A., Vila, M., Pérez, M.J., ... Castells, C. (2011). Assessment of a school-based intervention in eating habits and physical activity in school children: the AVall study. Journal Epidemiology Community Health, 65(10), 896-901. doi:10.1136/jech.2009.102319.

Marques, A., Gómez, F., Martins, J., Catunda, R., \& Sarmento, H. (2017). Association between physical education, school-based physical activity, and academic performance: a systematic review (Asociación entre la educación física, la actividad física en la escuela, y el rendimiento académico: una revisión sistemática). Retos, 31, 316-320.

Martínez Sabater, A., Marzá Gascón, A. Llorca Tauste, J., Martínez Puig, C., Escrivá Aznar, G., y Blasco Roque, M. (2013). Hábitos de salud en escolares en ámbito urbano y rural, Enfermería Global, 12(1), 158-169. Recuperado de http://cor.to/AATJ

Matthys, C., De Henauw, S., Bellemans, M., De Maeyer, M., \& De Backer, G. (2007). Breakfast habits affect overall nutrient profiles in adolescents. Public Health Nutrition, 10(4), 413-421.

Menal, S., Fajó, M. y Marques, I. (2011). Estudio descriptivo del desayuno en una población infantil inmigrante escolarizada, 2007-2010. Revista Española de Nutrición Humana y Dietética, 15(4), 177-183. doi:10.1016/S2173-1292(11)70057-6

Ministerio de Sanidad y Consumo (2005). Estrategia Naos. Estrategia para la nutrición, actividad física y prevención de la obesidad. Madrid: Agencia Española de Seguridad alimentaria. Recuperado de http://www.naos.aesan.msssi.gob.es/en/

Ministerio de Sanidad y Política Social, Ministerio de Educación (2007). Guía sobre obesidad infantil para profesionales sanitarios de atención primaria. Programa Perseo. Madrid: Ministerio de Sanidad y consumo. Recuperado de http://enfermeriacomunitaria.org/web/attachments/article/193/Guia_obesidad_infantil\%20para\%20Profesionales_sanitarios\%20de\%20AP.pdf

Ministerio de Sanidad, Política Social e Igualdad (2010). La alimentación de tus niños y niñas. Nutrición saludable de la infancia a la adolescencia. Madrid: Agencia Española de Seguridad Alimentaria y Nutrición (AESAN). Recuperado de http://www.aecosan. msssi.gob.es/AECOSAN/docs/documentos/nutricion/educanaos/alimentacion_ninos. pdf

Ministerio de Sanidad, Política Social e Igualdad (2011). Grupo de trabajo de la Guía de Práctica Clínica sobre Trastornos del Sueño en la Infancia y Adolescencia en Atención Primaria. Guía de Práctica Clínica sobre Trastornos del Sueño en la Infancia y Adolescencia en Atención Primaria. Plan de Calidad para el Sistema Nacional de 
Hábitos de alimentación, actividad física y horas de sueño en escolares: un estudio diagnóstico en Educación Primaria

Angustias González Rodríguez, Gabriel (H) Travé González y Francisca Maria Garcia

PADILLA

Salud del Ministerio de Sanidad, Política Social e Igualdad. Unidad de Evaluación de Tecnologías Sanitarias de la Agencia Laín Entralgo. Madrid: Ministerio de Ciencia e Innovación.

Molinero, O., Castro-Piñero, J., Ruiz, J.R., González-Montesinos, J.L., Mora, J., y Márquez, S. (2010). Conductas de salud en escolares de la provincia de Cádiz. Nutrición Hospitalaria, 25(2), 280-289. doi:10.3305/nh.2010.25.2.4579

Moreno, L.A., Gracia Marco, L. y Comité de Nutrición de la Asociación Española de Pediatría [CNAEP]. (2012). Prevención de la obesidad desde la actividad física: del discurso teórico a la práctica. Anales de Pediatría, 77(2), 136.e1-136.e6. doi:10.1016/j. anpedi.2012.04.011

Muñoz, A., Fernández, N. y Navarro, R. (2015). Estudio descriptivo sobre los hábitos saludables en alumnado de Primaria desde la educación física escolar. Sportis Scientific Technical Journal, 1 (1), 87-104.

Muñoz-Carrillo, J.C., Ruiz-Díaz, A., Hernández-Ruiz, A. y Córdoba-Ruiz, I. (2013). Las características del desayuno en alumnos de educación obligatoria. SPORT TK-Revista EuroAmericana de Ciencias del Deporte, 2(2), 45-49.

O'Donovan, G., Blazevich, A., Boreham, C., Cooper, A., Crank, H., Ekelund, U., ... Stamatakis, E. (2010). The ABC of Physical Activity for Health: a consensus statement from the British Association of Sport and Exercise Sciences. Journal Of Sports Sciences, 28(6), 573-591. doi:10.1080/02640411003671212

Organización Mundial de la Salud [OMS] (2010). Recomendaciones mundiales sobre actividad física para la salud. Ginebra: OMS. Recuperado de http://cor.to/AAZM

Organización Mundial de la Salud [OMS] (2017). Obesity and Overweight factsheet from the WHO. Health. Ginebra: OMS. Recuperado de http://www.thehealthwell. info/node/82914

Pozuelos, F. J., González, A., y Travé, G. (2008). Proyecto Curricular Investigando Nuestro Mundo (6-12). Investigando la alimentación humana. Sevilla: Díada.

Pozuelos, F.J., García-Prieto, F.J, y Gracia, F. J. (2017). Trabajo por proyectos para la promoción de la alimentación en la escuela en el medio rural. Aula de innovación educativa, (262), 43-48.

Paruthi, S., Brooks, L.J., D'Ambrosio, C., Hall, W.A., Kotagal, S., Lloyd, R.M., ... Rosen, C.L. (2016). Recommended amount of sleep for pediatric populations: a consensus statement of the American Academy of Sleep Medicine. Journal of clinical sleep medicine, 12(6), 785. doi:10.5664/jcsm.5866.

Poitras, V.J., Gray, C.E., Borghese, M.M., Carson, V., Chaput, J.P., Janssen, I., ... Sampson, M. (2016). Systematic review of the relationships between objectively measured physical activity and health indicators in school-aged children and youth. Applied Physiology, Nutrition, and Metabolism, 41(6), S197-S239. doi:10.1139/apnm-20150663.

Rampersaud, G.C., Pereira, M.A., Girard, B.L., Adams, J., y Metzl, J.D. (2005). RevieWbreakfast habits, nutricional status, body weight, and academic performance in children and adolescents. Journal of the American Dietetic Association, 105, 743-760. doi:10.1016/j.jada.2005.02.007

Sánchez-Carpintero, R. (2008). Trastornos del sueño en la niñez. En J. Narbona García y 
Hábitos de alimentación, actividad física y horas de sueño en escolares: un estudio diagnóstico en Educación Primaria

Angustias González Rodríguez, Gabriel (H) Travé González y Francisca Maria Garcia

PADILLA

C. Casas Fernández (Coords.) Protocolos Diagnóstico Terapeúticos de la AEP: Neurología Pediátrica (pp. 255-261). Asociación Española de Pediatría, Sociedad Española de Neurología Pediátrica. vol 34.

Schwartz, A. E., Leardo, M., Aneja, S., \& Elbel, B. (2016). Effect of a school-based water intervention on child body mass index and obesity. JAMA pediatrics, 170(3), 220-226. doi:10.1001/jamapediatrics.2015.3778

Serra, L. y Aranceta, J. (2004). Nutrición infantil y juvenil. Estudio enKid. Volumen 5. Barcelona: Masson.

Torres-García, M. y Santana-Hernández, H. (2017). La Educación para la Salud en la formación de maestros desde el Espacio Europeo de Educación Superior. Revista Complutense de Educación, 28(4), 1083-1101. doi:10.5209/RCED.51536

Tovar-Gálvez, M.I., Martín-Cuesta, M.A., González-Jiménez, E. y Schmidt-RioValle, J. (2017). Análisis sociodemográfico del estado y nivel nutricional y de actividad física de dos centros escolares de Granada (España). Journal of Negative and No Positive Results: JONNPR, 2(4), 152-159. doi:10.19230/jonnpr.1357

Uzhova, I., Fuster, V., Fernández-Ortiz, A., Ordovás, J.M., Sanz, J., Fernández-Friera, L., ... Peñalvo, J.L. (2017). The importance of breakfast in atherosclerosis disease: insights from the PESA study. Journal of the American College of Cardiology, 70(15), 18331842. doi:10.1016/j.jacc.2017.08.027

Varela, G. y Belmonte, S. (2015). El entorno escolar.En M. Rivero-Urgell, L.A. Moreno, J. Dalmau, J.M. Moreno, A. Aliaga, A. García-Perea., ... y J.M. Ávila (Coords.). El libro blanco de la alimentación escolar (pp. 305-311). Zaragoza: Prensas de la Universidad de Zaragoza.

Waters, E., Gibbs, L., Tadic, M., Ukoumunne, O.C., Magarey, A., Okely, A.D., ... Johnson, B. (2017). Cluster randomised trial of a school-community child health promotion and obesity prevention intervention: findings from the evaluation of fun ' $\mathrm{n}$ healthy in Moreland!. BMC public health, 18(1), 92. doi:10.1186/s12889-017-4625-9 
